practitioners know that today's wonder sedation is often tomorrow's fiasco. I do not know how many doctors require night sedation because of problems such as $\mathrm{Dr}$ Grant's, but I wonder how such doctors ever do any night work.

I was surprised that following an acute illness involving coronary care admission $\mathrm{Dr}$ Grant was allowed to travel to Malaysia three days later. I am not surprised that a few days later after landing in Malaysia he "fainted" and was again admitted to coronary care.

Dr Grant writes: "We, as doctors, are not doing nearly enough to relieve the psychological distress-the fear of death indeedwhich all intelligent patients with angina pectoris and coronary thrombosis are often allowed to suffer in a conspiracy of silence." Patients do not have to be intelligent to suffer such distress, and all general practitioners know this and deal with it all the time. Cardiac neurosis is a serious illness, and particularly so if patients are afraid to travel far, in case they go too far, by their way of thinking, from coronary care. There is much more to coronary thrombosis than coronary care, and sound advice and medical common sense are vital. I suggest that what $\mathrm{Dr}$ Grant requires is a general practitioner in every sense of the word, and he would be well advised to get one, seek his advice, and take it.

Musselburgh,

Clifford Lutton

Edinburgh EH21 6AG

\section{Photocoagulation and diabetic retinopathy}

SIR,-Absence overseas has prevented our commenting earlier on the excellent review article of $\mathrm{Mr}$ Hung Cheng (10 February, p 365) on "Photocoagulation and diabetic retinopathy." Proliferative change at the optic disc portends the worst possible prognosis, and yet initially often appears to remain in a quiescent state for years. ${ }^{1}$ Reluctance to institute treatment at this stage is a consequence of the false sense of security created by the apparent natural history of this phase in the disease. This "wait for progression" philosophy superficially appears to be vindicated by the degree of success (quoted by $\mathrm{Mr}$ Cheng) that we have demonstrated in photocoagulation during new-vessel progression, when the glial-tissue component of the proliferative complex is increasingly predominant."

Proliferative retinopathy is, however, a relentless, destructive process, constantly evolving, and involving a bipartite system of retinovitreal neovascularisation and vitreoretinal connective-tissue deposition which terminates in blindness, glaucoma, and retinal detachment. A misleadingly arbitrary subdivision into distinct phases is routinely made in clinical practice. This is an inevitable consequence of undue emphasis being laid on the rapidly advancing component of the proliferative process, which requires little subtle monitoring and lasts six to nine months.

Thus the generally accepted situation belies the true evolutionary characteristics of the retinopathy. A rigid adherence to a classification of $(a)$ initial quiescence, $(b)$ active, florid deterioration, (c) terminal cicatricial quiescence highlights the effects of the short intermediate phase of intense activity and omits the more subtle changes that do not manifest themselves as an obvious increase in the fibrovascular retinal pathology.
In our trial of therapeutic response to photocoagulation" those eyes which showed early peripapillary and epipapillary new vessels were subdivided into "active" and "quiescent" groups. Mr Cheng was correct in ascribing significantly better retention of vision in one of these subgroups (treated eyes). However, the "quiescent" group showed no difference up to three years. The group demonstrated the latency of onset of rapid deterioration and remained unchanged before treatment. Therapeutic benefits in such circumstances are slow in becoming evident, as the tendency to a fall in visual acuity is usually slower. However, a significantly beneficial response was clearly conferred on treated eyes when more subtle changes were monitored. In control eyes there were significantly more nerve-fibre bundle defects and greater deterioration in colour appreciation in the blue-green than in treated eyes.

It appears that early photocoagulation produces benefits in several ways. Firstly, it confers these benefits during an unobserved progression of the early "quiescent" phase of retinal neovascularisation. Secondly, several weeks may be required in which to complete treatment, and if the disease is active there is a potentially blinding condition which is increasing through the period of photocoagulation. Any prevarication and delay until observed "activity" presents inevitably leads to treatment benefits being conferred too late, or else vitreous haemorrhage makes photocoagulation impossible at the vital time. Finally, in the rationale of "early" versus "wait for progression" photocoagulation the quintessence of treatment benefit is to be found as much in the attitude of mind of those who embrace a change to prompt and early treatment as in the modalities and techniques they adopt.

Manchester Royal Eye Hospital,

B L Hercules Manchester 13

I I GAYED

Ahmedi Hospital

Ahmedi, Kuwait

'Larsen, H W C, Acta Ophthalmologica, 1960, suppl No $60, \mathrm{p} 42$. Hercules, B L, et al, British fournal of ()phthalmology,
1977, 61, 555 .

\section{Diagnosis of cholestasis}

SIR,-We would like to comment on your recent leading article (12 May, p 1232). Your editorial took exception to our published statement ${ }^{1}$ that it may be unnecessary to confirm (by preoperative cholangiography) that jaundice is caused by gall stones when ultrasonography shows only stones in the gall bladder.

Of course, this recommendation is made only when there are no clinical or ultrasonographic features to suggest an alternative cause for jaundice, and further extensive experience has not changed our opinion. However, it is true that most jaundiced patients do need invasive cholangiography even after expert ultrasonography. The relative merits of percutaneous transhepatic cholangiography (PTC) and endoscopic retrograde cholangiopancreatography (ERCP) have been well rehearsed, but we wish to emphasise two points.

Firstly, the results and risks of both techniques are highly dependent on the experience of the operator. In the learning phase both methods carry a significant failure rate and complication rate. ${ }^{2}{ }^{3}$ Our success rate for endoscopic cholangiography is now well over $90 \%$ and we have not seen a complication in the last 1000 diagnostic ERCP examinations.

Secondly, the choice of cholangiography route is increasingly being influenced by the therapeutic applications. Thus we may well choose PTC to confirm a clinical and ultrasonographic diagnosis of malignant obstructive jaundice, in order to insert a drainage tube or endoprosthesis at the same time; we choose ERCP in many patients who probably have stones because it allows us at the same time to remove any duct stones by endoscopic sphincterotomy.

P B CotToN

Gastrointestinal Unit,

W R LEES

Department of Radiology,

Middlesex Hospital

' Vallon, A G, Lees, W R, and Cotton, P B, Gut, 1979, 20, 51 .

Juler, G L, Conroy, R M, and Fuelleman, R W, Archives of Surgery, 1977, 112, 954.
${ }^{3}$ Bilbao, M K, et al, Gastroenterology, 1976, 70, 314.

\section{Operative cholangiography}

SIR,-At this hospital operative cholangiography is routine during cholecystectomy. The operating table can be tilted and it has a fixed grid incorporated within it. However, the films have often been technically disappointing. The problem was the difficulty in striking a balance between the need to tilt the table in order to display the common bile duct clear of the spine and tilting too much so that grid "cut-off" gave rise to uneven exposure and poor definition.

The grid lines were at that time aligned along the long axis of the operating table. We have solved the problem quite simply by removing the grid and rotating it through $90^{\circ}$ so that the grid lines are now aligned across the table. When the table is tilted obliquely grid cut-off cannot now occur. In addition, we routinely use a cone on the tube head in order to reduce scatter and this also improves radiographic definition.

These changes have transformed the quality of our operative radiography and are recommended to any theatre with a similar table where similar difficulties have occurred.

BRIAN W ELLIS AlaN G Cox ROBERT WILKINS GERALD DE LACEY

\section{Departments of Surgery and Diagnostic Radiology, Northwick Park Hospital an Clinical Research Centre,}

\section{Tap water for electrocardiographic} recording

SIR,-It was with great interest that I read the article (17 February, p 454) from the Charitable Infirmary, Dublin, on substituting tapwater for electrode jelly. It is most laudable that this attempt to reduce expenses has been made.

I would like to note that the use of surgical spirit $B P C$ is just as good as water. I have personally used this method since I learnt about its use from Professor A Nadas at the Children's Medical Centre, Cardiac Clinic, Boston, in 1961. The ECG recordings are as 